\title{
LINEAR FINITE ELEMENT METHODS FOR PLANAR LINEAR ELASTICITY
}

\author{
SUSANNE C. BRENNER AND LI-YENG SUNG
}

\begin{abstract}
A linear nonconforming (conforming) displacement finite element method for the pure displacement (pure traction) problem in two-dimensional linear elasticity for a homogeneous isotropic elastic material is considered. In the case of a convex polygonal configuration domain, $\mathscr{O}(h)\left(\mathscr{O}\left(h^{2}\right)\right)$ error estimates in the energy $\left(L^{2}\right)$ norm are obtained. The convergence rate does not deteriorate for nearly incompressible material. Furthermore, the convergence analysis does not rely on the theory of saddle point problems.
\end{abstract}

\section{INTRODUCTION}

We consider finite element approximations of the pure displacement and pure traction boundary value problems in two-dimensional linear elasticity associated with a homogeneous isotropic elastic material.

It is well known (cf. [5]) that the convergence rate for the standard displacement method using continuous linear finite elements deteriorates as the Lamé constant $\lambda$ becomes large, i.e., when the elastic material is nearly incompressible. Various methods have been proposed which work uniformly well for all $\lambda$, for example the $p$-version method of Vogelius in [22], the PEERS method of Arnold, Brezzi, and Douglas in [1], the mixed method of Stenberg in [20], the Galerkin least squares method of Franca and Stenberg in [11], and the nonconforming methods of Falk in [10]. A common theme in these works is that the convergence analysis is reduced to the study of the stability condition for a saddle point problem.

Here we will directly prove (without referring to any saddle point problem) the uniform convergence with respect to $\lambda$ of two displacement methods using conforming and nonconforming finite elements. What we need in the convergence proof is an interpolation operator $\Pi$ with the property $(P)$ : $\operatorname{div} \phi=0 \Rightarrow \operatorname{div} \Pi \phi=0$. Such an operator does not exist for the conforming linear finite element space, which explains the deterioration of the convergence rate. This difficulty can be overcome in two ways. First, one can enlarge the finite element space by using nonconforming finite elements, which is the approach that we adopt for the pure displacement problem. Second, one can keep

Received by the editor January 14, 1991 and, in revised form, November 26, 1991.

1991 Mathematics Subject Classification. Primary 65N30, 73V05.

Key words and phrases. Linear finite elements, linear elasticity.

The work of the first author was supported in part by the National Science Foundation under Grant No. DMS-90-96126. 
the conforming linear finite element space, but use reduced integration in the formulation of the discretized equations, so that $(\mathrm{P})$ only needs to hold in a weaker sense. This is the approach that we take for the pure traction problem. In both cases we obtain uniform $\mathscr{O}(h)$ energy norm estimates for the discretization error, and for the pure displacement problem we also obtain a uniform $\mathscr{O}\left(h^{2}\right) L^{2}$-norm estimate. In our proof we also use some properties of the divergence operator developed by Arnold, Scott, and Vogelius in [2]. Our methods are robust in the sense of Babuška and Suri (cf. [3, 4]).

In order to write down the equations of the boundary value problems, we introduce the following notation. Throughout this paper, an undertilde is used to denote vector-valued functions, operators, and their associated spaces. Double undertildes are used for matrix-valued functions and operators. We define

$$
\begin{gathered}
\underset{\sim}{\operatorname{grad} p}=\left(\begin{array}{l}
\partial p / \partial x_{1} \\
\partial p / \partial x_{2}
\end{array}\right), \quad \underset{\sim}{\operatorname{div} \underset{\approx}{\tau}=}=\left(\begin{array}{l}
\partial \tau_{11} / \partial x_{1}+\partial \tau_{12} / \partial x_{2} \\
\partial \tau_{21} / \partial x_{1}+\partial \tau_{22} / \partial x_{2}
\end{array}\right), \\
\operatorname{curl} p=\left(\begin{array}{c}
\partial p / \partial x_{2} \\
-\partial p / \partial x_{1}
\end{array}\right), \quad \operatorname{div} \underset{\sim}{v}=\partial v_{1} / \partial x_{1}+\partial v_{2} / \partial x_{2}, \\
\operatorname{rot} v=-\partial v_{1} / \partial x_{2}+\partial v_{2} / \partial x_{1}, \quad \underset{\sim}{\operatorname{grad}} \underset{\sim}{v}=\left(\begin{array}{ll}
\partial v_{1} / \partial x_{1} & \partial v_{1} / \partial x_{2} \\
\partial v_{2} / \partial x_{1} & \partial v_{2} / \partial x_{2}
\end{array}\right) .
\end{gathered}
$$

We also define

$$
\underset{\approx}{\delta}=\left(\begin{array}{ll}
1 & 0 \\
0 & 1
\end{array}\right) \quad \text { and } \quad \operatorname{tr}(\underset{\approx}{\tau})=\underset{\approx}{\tau}: \underset{\approx}{\delta}
$$

where

$$
\underset{\approx}{\sigma}: \underset{\approx}{\tau}=\sum_{i=1}^{2} \sum_{j=1}^{2} \sigma_{i j} \tau_{i j} .
$$

Finally,

$$
\underset{\sim}{\varepsilon}(\underset{\sim}{v})=\frac{1}{2}\left[\underset{\sim}{\operatorname{grad}} \underset{\sim}{v}+(\underset{\sim}{\operatorname{grad}} \underset{\sim}{v})^{t}\right]
$$

The pure displacement boundary value problem for a two-dimensional homogeneous isotropic material is given by

$$
\begin{aligned}
& -\underset{\sim}{\operatorname{div}}\{2 \mu \underset{\sim}{\varepsilon} \underset{\sim}{\mathcal{u}})+\lambda \operatorname{tr}(\underset{\sim}{\varepsilon} \underset{\sim}{\mathcal{u}})) \underset{\approx}{\delta}\}=\underset{\sim}{f} \text { in } \Omega, \\
& \underset{\sim}{u}=\underset{\sim}{g} \text { on } \partial \Omega,
\end{aligned}
$$

where $\Omega \subseteq \mathbb{R}^{2}$ is the configuration domain, $\underset{\sim}{u}$ is the displacement, $f(x)$ is the body force, and $\lambda, \mu>0$ are the Lamé constants. The pure traction problem is given by

$$
\begin{aligned}
& -\underset{\operatorname{div}}{\sin }\{\underset{\sim}{\boldsymbol{\varepsilon}}(\underset{\sim}{u})+\lambda \operatorname{tr}(\underset{\sim}{\varepsilon} \underset{\sim}{(u)}) \underset{\approx}{\delta}\}=\underset{\sim}{f} \text { in } \Omega, \\
& (2 \mu \underset{\sim}{\varepsilon} \underset{\sim}{u})+\lambda \operatorname{tr}(\underset{\sim}{\varepsilon} \underset{\sim}{(u)}) \underset{\approx}{\delta}) \underset{\sim}{\nu}=\underset{\sim}{g} \text { on } \partial \Omega,
\end{aligned}
$$

where $\nu$ is the unit outer normal. For simplicity, we only consider the case where $\tilde{\Omega}$ is a bounded convex polygonal domain throughout this paper. 
In the treatment of the pure traction problem we need the spaces $(k \geq 1)$

$$
\widehat{\widehat{H}}^{k}(\Omega)=\left\{\underset{\sim}{v} \in \underset{\sim}{H^{H}}(\Omega): \int_{\Omega} \underset{\sim}{v} d x=\underset{\sim}{0}, \int_{\Omega} \operatorname{rot} \underset{\sim}{v} d x=0\right\} .
$$

We also use the following conventions for the Sobolev norms and seminorms:

$$
\begin{aligned}
& \|\underset{\sim}{v}\|_{H^{m}(\Omega)}:=\left(\int_{\Omega} \sum_{|\alpha| \leq m}\left|\partial^{\alpha} \underset{\sim}{v}\right|^{2} d x\right)^{1 / 2}, \\
& \mid \underset{\sim}{\mid v}{\underset{\sim}{H^{m}(\Omega)}}:=\left(\int_{\Omega} \sum_{|\alpha|=m}\left|\partial^{\alpha} \underset{\sim}{v}\right|^{2} d x\right)^{1 / 2} .
\end{aligned}
$$

The rest of the paper is organized as follows. In $\S 2$ we give the relevant results from the theory of partial differential equations on polygonal domains. We prove in Lemmas 2.2 and 2.3 that there exist uniform elliptic regularity estimates for $\|\underset{\sim}{u}\|_{H^{2}(\Omega)}+\lambda\|\operatorname{div} \underset{\sim}{u}\|_{H^{1}(\Omega)}$ for the boundary value problems (1.1) and (1.2). To the best of our knowledge, these estimates have not appeared explicitly in the existing literature in the context of convex polygonal domains. Section 3 contains a discussion of the pure displacement problem, and $\S 4$ contains a discussion of the pure traction problem.

\section{RELEVANT RESULTS FROM THE THEORY OF PARTIAL DIFFERENTIAL EQUATIONS ON POLYGONAL DOMAINS}

We consider the Lamé constants $(\mu, \lambda)$ in the range $\left[\mu_{0}, \mu_{1}\right] \times(0, \infty)$, where $0<\mu_{0}<\mu_{1}<\infty$. We let $C$ denote a generic positive constant, independent of $\mu$ and $\lambda$, with its dependencies listed as its subscripts. The same symbol may take different values in different contexts.

We begin with some properties of the divergence operator.

Lemma 2.1. Let $\Omega \subset \mathbb{R}^{2}$ be any bounded polygonal domain and let $l=1$ or 2. There exists a positive constant $C_{\Omega}$ such that given any $\underset{\sim}{v} \underset{\sim}{H_{\sim}^{l}}(\Omega) \cap \underset{\sim}{H_{0}^{1}}(\Omega)$ (respectively, $\underset{\sim}{v} \in \underset{\sim}{H^{l}}(\Omega)$ ), there exists $\underset{\sim}{w} \in \underset{\sim}{H^{l}}(\Omega) \cap \underset{\sim}{H}(\Omega)$ (respectively, $\underset{\sim}{w} \in$ $\left.\widehat{\sim}^{l}(\Omega)(c f .(1.3))\right)$ such that

$$
\operatorname{div} \underset{\sim}{w}=\operatorname{div} \underset{\sim}{v}
$$

and

$$
\|\underset{\sim}{w}\|_{H^{l}(\Omega)} \leq C_{\Omega}\|\operatorname{div} \underset{\sim}{v}\|_{H^{l-1}(\Omega)} .
$$

Proof. If $\underset{\sim}{v} \in{\underset{\sim}{H}}^{l}(\Omega) \cap \underset{\sim}{H_{0}^{1}}(\Omega)$, then the existence of $\underset{\sim}{w}$ with properties $(2.1)$ and (2.2) follows from Theorem 3.1 and the inclusion (3.2) in [2]. It should be noted that the analysis for this case is much deeper than the following simple argument for the second case.

If $\underset{\sim}{v} \in \underset{\sim}{H^{l}}(\Omega)$, then $\operatorname{div} \underset{\sim}{v} \in{\underset{\sim}{H}}^{l-1}(\Omega)$. Let $D$ be an open disc that contains $\bar{\Omega}$. There exists an extension operator (cf. [18]) $E: H^{l-1}(\Omega) \rightarrow H^{l-1}(D)$ such that

$$
\|E(q)\|_{H^{l-1}(D)} \leq C_{\Omega}\|q\|_{H^{l-1}(\Omega)} \quad \forall q \in H^{l-1}(\Omega)
$$


and

$$
\left.E(q)\right|_{\Omega}=q \quad \forall q \in H^{l-1}(\Omega) .
$$

Let $\zeta \in H^{l+1}(\Omega)$ be the solution of

$$
\begin{array}{cl}
\Delta \zeta=E(\operatorname{div} \underset{\sim}{v}) & \text { in } D, \\
\zeta=0 & \text { on } \partial D .
\end{array}
$$

Then from elliptic regularity (cf. [18]) we have

$$
\|\zeta\|_{H^{l+1}(D)} \leq C_{\Omega}\|E(\operatorname{div} \underset{\sim}{v})\|_{H^{l-1}(D)}
$$

Let

$$
\left.\underset{\sim}{w} \underset{\sim}{\operatorname{grad}} \zeta\right|_{\Omega}-\frac{1}{|\Omega|} \int_{\Omega} \underset{\operatorname{grad}}{\sim} d x .
$$

It is clear that $\underset{\sim}{w} \in \underset{\sim}{\widehat{H}^{l}}(\Omega)$. Properties (2.1) and (2.2) then follow from (2.3)(2.6).

Since the boundary of a polygon has corners, the boundary conditions in (1.1) and (1.2) must be carefully interpreted. We shall denote by $S_{i}, 1 \leq i \leq n$, the vertices of $\Omega$, by $\Gamma_{i}, 1 \leq i \leq n$, the open line segments joining $S_{i}$ to $S_{i+1}$, by $\underset{\sim}{\tau_{i}}$ the positively oriented unit tangent along $\Gamma_{i}$, and by $\underset{\sim}{\nu_{i}}$ the unit outer normal along $\Gamma_{i}$. Henceforth, indices involving the vertices and edges of $\Omega$ should be interpreted as integers modulo $n$.

Let $p \in H^{1 / 2}\left(\Gamma_{i}\right)$ and $q \in H^{1 / 2}\left(\Gamma_{i+1}\right)$. We say that $p \equiv q$ at $S_{i+1}$ if $\int_{0}^{\delta}|q(s)-p(-s)|^{2} \frac{d s}{s}<\infty$, where $s$ is the oriented arc length measured from $S_{i+1}$, and $\delta$ is a positive number less than $\min \left\{\left|\Gamma_{i}\right|: 1 \leq i \leq n\right\}$.

Lemma 2.2. Let $\Omega \subseteq \mathbb{R}^{2}$ be a bounded convex polygonal domain, $\underset{\sim}{f} \in \underset{\sim}{L^{2}}(\Omega)$, $\underset{\sim}{g_{i}} \in \underset{\sim}{H / 2}\left(\Gamma_{i}\right)$, and $\underset{\sim}{g_{i}}\left(S_{i+1}\right)=\underset{\sim}{g}{ }_{i+1}\left(S_{i+1}\right)$ for $1 \leq i \leq n$. Then the pure displacement problem

$$
\begin{aligned}
&-\operatorname{div}\{2 \mu \underset{\approx}{\varepsilon} \underset{\sim}{u})+\lambda \operatorname{tr}(\underset{\approx}{\varepsilon(\underset{\sim}{u}))} \underset{\approx}{\delta}\}=\underset{\sim}{f} \quad \text { in } \Omega, \\
&\left.\underset{\sim}{u}\right|_{\Gamma_{i}}=g_{\sim} \quad \text { for } 1 \leq i \leq n
\end{aligned}
$$

has a unique solution $\underset{\sim}{u} \in \underset{\sim}{H^{2}}(\Omega)$. If we assume further that $\sum_{i=1}^{n} \int_{\Gamma_{i}} \underset{\sim}{g_{i}} \cdot \underset{\sim}{\nu_{i}} d s=$ 0 , and

$$
\frac{\partial}{\partial \tau_{i}} \underset{\sim}{g_{i}} \cdot \underset{\sim}{\nu_{i+1}} \equiv \frac{\partial}{\partial \tau_{i+1}} \underset{\sim}{g_{i+1}} \cdot \underset{\sim}{\nu_{i}}
$$

at $S_{i+1}$ for $1 \leq i \leq n$, then there exists a positive constant $C_{\Omega}$ such that

$$
\|\underset{\sim}{u}\|_{H^{2}(\Omega)}+\lambda\|\operatorname{div} \underset{\sim}{u}\|_{H^{1}(\Omega)} \leq C_{\Omega}\left\{\|\underset{\sim}{\|}\|_{L^{2}(\Omega)}+\sum_{i=1}^{n}\|\underset{\sim}{g}\|_{H^{3 / 2}\left(\Gamma_{i}\right)}\right\} .
$$

Proof. The existence, uniqueness, and smoothness of the solution (2.7) are well known (cf. [14, 18, 21]). Here we will indicate how the estimate (2.8) can be obtained.

Observe that the conditions on $g_{i}$ are equivalent to (cf. [2]) the existence of a function $z \in H^{3}(\Omega)$ such that $\left.\underline{\operatorname{curl} z}\right|_{\Gamma_{1}}=g_{i}$ for $1 \leq i \leq n$, which 
is a necessary condition for $(2.8)$ to hold for all $\lambda \in(0, \infty)$. Moreover, it follows from Theorem 6.2 of [2] that $z$ can be chosen with the property that $\|z\|_{H^{3}(\Omega)} \leq C_{\Omega} \sum_{i=1}^{n}\left\|g_{i}\right\|_{H^{3 / 2}\left(\Gamma_{i}\right)}$. Therefore, it suffices to establish (2.8) for $\underset{\sim}{u}$ - curl $z$, or equivalently, we may assume $g_{i}=\underset{\sim}{0}$ without loss of generality. In other words, we assume that $\underset{\sim}{u} \in \underset{\sim}{H^{2}}(\Omega) \cap \underset{\sim}{H_{0}^{1}}(\Omega)$ satisfies

$$
-\underset{\sim}{\operatorname{div}}\{2 \mu \underset{\sim}{\varepsilon}(\underset{\sim}{u})+\lambda \operatorname{tr}(\underset{\sim}{\varepsilon(u)} \underset{\sim}{u} \underset{\approx}{\delta}\}=\underset{\sim}{f} \text { in } \Omega .
$$

We first show that there exists a positive constant $C_{\Omega}$ such that

$$
\|\underset{\sim}{u}\|_{H^{1}(\Omega)}+\lambda\|\operatorname{div} \underset{\sim}{u}\|_{L^{2}(\Omega)} \leq C_{\Omega}\|\underset{\sim}{f}\|_{L^{2}(\Omega)} .
$$

Let $\underset{\sim}{w} \in \underset{\sim}{H_{0}^{1}}(\Omega)$. By integration by parts, it follows from (2.9) that

$$
\left.2 \mu \int_{\Omega} \underset{\sim}{\varepsilon} \underset{\sim}{u}\right): \underset{\sim}{\varepsilon}(\underset{\sim}{w}) d x+\lambda \int_{\Omega}(\operatorname{div} \underset{\sim}{u})(\operatorname{div} \underset{\sim}{w}) d x=\int_{\Omega} \underset{\sim}{f} \cdot \underset{\sim}{w} d x .
$$

If we let $\underset{\sim}{w}=\underset{\sim}{u}$ in $(2.11)$, we obtain

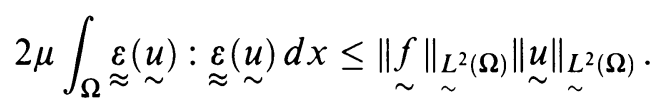

By using Korn's first inequality and Poincaré's inequality (cf. $[12,18]$ ), we have

$$
\|\underset{\sim}{u}\|_{H^{1}(\Omega)} \leq C_{\Omega}\|\underset{\sim}{f}\|_{L^{2}(\Omega)} .
$$

By Lemma 2.1, there exists $\underset{\sim}{w^{*}} \in \underset{\sim}{H_{0}^{1}}(\Omega)$ such that

$$
\operatorname{div} \underset{\sim}{w^{*}}=\operatorname{div} \underset{\sim}{u}
$$

and

$$
\left\|\underset{\sim}{w^{*}}\right\|_{H^{1}(\Omega)} \leq C_{\Omega}\|\operatorname{div} \underset{\sim}{u}\|_{L^{2}(\Omega)} .
$$

It follows from (2.11) and (2.14) that

$$
\begin{aligned}
& \lambda \int_{\Omega} \mid \operatorname{div} \underset{\sim}{\left.\underset{\sim}{u}\right|^{2}} d x \leq\|\underset{\sim}{f}\|_{L^{2}(\Omega)}\left\|w_{\sim}^{*}\right\|_{L^{2}(\Omega)} \\
& +2 \mu\|\underset{\approx}{\underset{\sim}{\varepsilon}(u)}\|_{L^{2}(\Omega)}\left\|\underset{\approx}{\varepsilon} \underset{\sim}{\left.w^{*}\right)}\right\|_{L^{2}(\Omega)} .
\end{aligned}
$$

Together with (2.13) and (2.15), we have

$$
\lambda\|\operatorname{div} \underset{\sim}{u}\|_{L^{2}(\Omega)} \leq C_{\Omega}\|\underset{\sim}{f}\|_{L^{2}(\Omega)} .
$$

The estimate (2.10) now follows from (2.13) and (2.17).

Next we will show that there exists a positive constant $C_{\Omega}$ such that

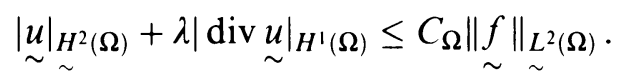

Observe that it suffices to prove (2.18) for $\lambda>\lambda_{0}$, where $\lambda_{0}$ is a sufficiently large number. (The case $\lambda \leq \lambda_{0}$ follows directly from standard elliptic regularity estimates for (2.9).) 
From Lemma 2.1, there exists $\phi \in \underset{\sim}{H^{2}}(\Omega) \cap \underset{\sim}{H_{0}^{1}}(\Omega)$ such that

$$
\operatorname{div} \underset{\sim}{\phi}=\operatorname{div} \underset{\sim}{u}
$$

and

$$
\|\underset{\sim}{\phi}\|_{H^{2}(\Omega)} \leq C_{\Omega}\|\operatorname{div} \underset{\sim}{u}\|_{H^{1}(\Omega)}
$$

Observe that equation (2.7) can be rewritten as

$$
-\mu \underset{\sim}{u}-(\mu+\lambda) \underset{\sim}{\operatorname{grad}}(\operatorname{div} \underset{\sim}{u})=f .
$$

Define

$$
\underset{\sim}{u^{\prime}}:=\underset{\sim}{u}-\underset{\sim}{\phi} \text { and } p:=-\left(\frac{\mu+\lambda}{\mu}\right) \operatorname{div} \underset{\sim}{u} \text {. }
$$

Then $\left.\left(\underset{\sim}{u^{\prime}}, p\right) \in \underset{\sim}{H^{2}}(\Omega) \cap \underset{\sim}{H}(\Omega)\right) \times \underset{\sim}{H^{1}}(\Omega)$ satisfies the following Stokes equations in $\Omega$ :

$$
\begin{aligned}
-\Delta \underset{\sim}{u^{\prime}}+\underset{\sim}{\operatorname{grad}} p & =\frac{1}{\mu} f+\Delta \phi, \\
\operatorname{div} \underset{\sim}{u^{\prime}} & =0 .
\end{aligned}
$$

Observe that by using (2.13) and (2.20) we have

$$
\|\underset{\sim}{\phi}\|_{H^{2}(\Omega)} \leq C_{\Omega}\left(|\operatorname{div} \underset{\sim}{u}|_{H^{1}(\Omega)}+\|\underset{\sim}{f}\|_{L^{2}(\Omega)}\right) \text {. }
$$

By Theorem 2 in [16],

$$
\left\|\underset{\sim}{u_{\sim}^{\prime}}\right\|_{H^{2}(\Omega)}+|p|_{H^{1}(\Omega)} \leq C_{\Omega}\left\{\frac{1}{\mu}\|\underset{\sim}{f}\|_{L^{2}(\Omega)}+\|\underset{\sim}{\Delta}\|_{L^{2}(\Omega)}\right\} .
$$

Substituting (2.22) into (2.25) and applying (2.24) yields

$$
\|\underset{\sim}{u}\|_{H^{2}(\Omega)}+\frac{\mu+\lambda}{\mu}|\operatorname{div} \underset{\sim}{u}|_{H^{1}(\Omega)} \leq C_{\Omega}\left\{\|\underset{\sim}{f}\|_{L^{L^{2}(\Omega)}}+\mid \operatorname{div} \underset{\sim}{\left.u\right|_{H^{1}(\Omega)}}\right\} .
$$

Let $\lambda_{0}=2 C_{\Omega} \mu_{1}$, where $C_{\Omega}$ is the constant in (2.26). For $\lambda>\lambda_{0}$, we obtain from (2.26) that

$$
\|\underset{\sim}{u}\|_{H^{2}(\Omega)}+\frac{\lambda}{2 \mu_{1}}|\operatorname{div} \underset{\sim}{u}|_{H^{1}(\Omega)} \leq C_{\Omega}\|\underset{\sim}{f}\|_{H^{2}(\Omega)},
$$

which implies (2.18) for $\lambda>\lambda_{0}$.

We now turn to the pure traction problem. For this problem to be solvable, certain compatibility conditions must be satisfied. For that purpose we introduce the space of infinitesimal rigid motions $\mathbf{R M}:=\left\{\underset{\sim}{v:}{\underset{\sim}{t}}^{t}=\left(a+b x_{2}\right.\right.$, $\left.\left.c-b x_{1}\right), a, b, c \in \mathbb{R}\right\}$.

Lemma 2.3. Let $\Omega \subseteq \mathbb{R}^{2}$ be a bounded convex polygonal domain. Let $\underset{\sim}{f} \in$

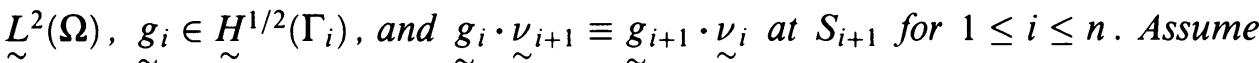
the following compatibility condition to hold:

$$
\int_{\Omega} \underset{\sim}{f} \cdot \underset{\sim}{v} d x+\sum_{i=1}^{n} \int_{\Gamma_{i}} \underset{\sim}{g_{i}} \cdot \underset{\sim}{v} d s=0 \quad \forall \underset{\sim}{v} \in \mathbf{R} \mathbf{M} .
$$


Then the pure traction problem

$$
\begin{aligned}
& -\operatorname{div}\{2 \mu \underset{\approx}{\varepsilon}(\underset{\sim}{u})+\lambda \operatorname{tr}(\underset{\sim}{\varepsilon} \underset{\sim}{u})) \underset{\approx}{\delta}\}=f_{\sim} \quad \text { in } \Omega, \\
& (2 \mu \underset{\approx}{\varepsilon}(u)+\lambda \operatorname{tr}(\underset{\sim}{\varepsilon} \underset{\sim}{u})) \underset{\approx}{\operatorname{d}})\left.\nu_{i}\right|_{\Gamma_{i}}=g_{\sim}, \quad 1 \leq i \leq n,
\end{aligned}
$$

has a unique solution $\underset{\sim}{u} \in \widehat{\sim}_{\sim}{ }^{2}(\Omega)(c f .(1.3))$. Moreover, there exists a positive constant $C_{\Omega}$ such that

$$
\|\underset{\sim}{u}\|_{H^{2}(\Omega)}+\lambda \| \operatorname{div} \underset{\sim}{\underset{\sim}{u} \|_{H^{1}(\Omega)} \leq C_{\Omega}}\left\{\|\underset{\sim}{f}\|_{L^{2}(\Omega)}+\sum_{i=1}^{n}\left\|\underset{\sim}{g_{\sim}}\right\|_{H^{1 / 2}\left(\Gamma_{i}\right)}\right\} .
$$

Proof. The existence, uniqueness, and smoothness of the solution of (2.29) are well known (cf. $[14,15,18,21]$ ). It remains to establish the estimate (2.30). In the case of a domain with smooth boundary, this estimate was proved by Vogelius in [22].

First we show that it suffices to establish (2.30) in the case $f=\underset{\sim}{0}$. that

$$
-\operatorname{div}\{2 \mu \underset{\sim}{\varepsilon} \underset{\sim}{w})+\lambda \operatorname{tr}(\underset{\sim}{\varepsilon} \underset{\sim}{w})) \underset{\sim}{\delta}\}=\underset{\sim}{f} \text { in } \Omega
$$

and

$$
\|\underset{\sim}{w}\|_{H^{2}(\Omega)}+\lambda\|\operatorname{div} \underset{\sim}{w}\|_{H^{1}(\Omega)} \leq C_{\Omega}\|\underset{\sim}{f}\|_{L^{2}(\Omega)} .
$$

Let $(a, c)^{t}=\int_{\Omega} \underset{\sim}{w} d x, b=\frac{1}{2} \int_{\Omega} \operatorname{rot} \underset{\sim}{w} d x$, and

$$
{\underset{\sim}{w}}^{*}=\underset{\sim}{w}+\left(-a+b x_{2},-c-b x_{1}\right)^{t}
$$

Then $\underset{\sim}{w^{*}} \in \widehat{H}^{2}(\Omega)$ such that

$$
\left.\left.-\operatorname{div}\left\{2 \mu \underset{\sim}{\varepsilon} \underset{\sim}{w^{*}}\right)+\lambda \operatorname{tr}\left(\underset{\sim}{\varepsilon} \underset{\sim}{w^{*}}\right)\right) \underset{\approx}{\delta}\right\}=\underset{\sim}{f} \text { in } \Omega
$$

and

$$
\left\|\underset{\sim}{w^{*}}\right\|_{H^{2}(\Omega)}+\lambda\left\|\operatorname{div} \underset{\sim}{w^{*}}\right\|_{H^{1}(\Omega)} \leq C_{\Omega}\|\underset{\sim}{f}\|_{L^{2}(\Omega)}
$$

Let

$$
\left.\left.\left.\underset{\sim}{q_{i}}=\left(2 \mu \underset{\sim}{\varepsilon} \underset{\sim}{w^{*}}\right)+\lambda \operatorname{tr}\left(\underset{\sim}{\varepsilon} \underset{\sim}{\left(w^{*}\right.}\right)\right) \underset{\sim}{\delta}\right)\left.\underset{\sim}{v_{i}}\right|_{\Gamma_{i}}=2 \mu \underset{\sim}{\varepsilon} \underset{\sim}{\left(w^{*}\right.}\right)\left.\underset{\sim}{v_{i}}\right|_{\Gamma_{i}}+\left.\lambda\left(\operatorname{div} \underset{\sim}{w^{*}}\right) v_{i}\right|_{\Gamma_{i}} .
$$

From the trace theorem and (2.35), we have

$$
\|\underset{\sim}{q}\|_{H^{1 / 2}\left(\Gamma_{i}\right)} \leq C_{\Omega}\|\underset{\sim}{f}\|_{L^{2}(\Omega)}
$$

In view of the estimates (2.36), we may instead establish (2.30) for $\underset{\sim}{u}-\underset{\sim}{w^{*}}$. In other words, there is no loss of generality if we assume $\underset{\sim}{f}=\underset{\sim}{0}$ in $(2.29)$.

The compatibility condition (2.28) now becomes

$$
\sum_{i=1}^{n} \int_{\Gamma_{i}} \underset{\sim}{g_{i}} d s=\underset{\sim}{0} \text { and } \sum_{i=1}^{n} \int_{\Gamma_{i}}\left(x_{2} g_{i 1}-x_{1} g_{i 2}\right) d s=0 .
$$


Using (2.37), we can define (cf. [15, §2]) $\underset{\sim}{\xi_{i}}, h_{i}$, and $k_{i}$ such that $\underset{\sim}{\xi_{1}}\left(S_{1}\right)=\underset{\sim}{0}$, $h_{1}\left(S_{1}\right)=0$, and for $1 \leq i \leq n$,

$$
\underset{\sim}{\xi_{i}}\left(S_{i+1}\right)={\underset{\sim}{i+1}}_{\xi_{i+1}}\left(S_{i+1}\right), \quad h_{i}\left(S_{i+1}\right)=h_{i+1}\left(S_{i+1}\right),
$$

and

$$
\frac{\partial}{\partial \tau_{i}} \xi_{i 1}=-g_{i 2}, \quad \frac{\partial}{\partial \tau_{i}} \xi_{i 2}=g_{i 1}, \quad k_{i}=\underset{\sim}{\xi_{i}} \cdot \underset{\sim}{\nu_{i}}, \quad \frac{\partial}{\partial \tau_{i}} h_{i}=\underset{\sim}{\xi_{i}} \cdot \underset{\sim}{\tau_{i}} .
$$

It follows that $\xi_{i} \in H^{3 / 2}\left(\Gamma_{i}\right), k_{i} \in H^{3 / 2}\left(\Gamma_{i}\right)$, and $h_{i} \in H^{5 / 2}\left(\Gamma_{i}\right)$. Moreover, we have

$$
\sum_{i=1}^{n}\left(\left\|k_{i}\right\|_{H^{3 / 2}\left(\Gamma_{i}\right)}+\left\|h_{i}\right\|_{H^{5 / 2}\left(\Gamma_{i}\right)} \leq C_{\Omega} \sum_{i=1}^{n}\left\|g_{i}\right\|_{H^{1 / 2}\left(\Gamma_{i}\right)} .\right.
$$

Using the symmetry of $\{2 \mu \underset{\sim}{\underset{\sim}{\varepsilon}}(\underset{\sim}{u})+\lambda(\operatorname{tr} \underset{\sim}{\underset{\sim}{\varepsilon}} \underset{\sim}{u})) \underset{\sim}{\delta}\}$ and (2.29) with $\underset{\sim}{f}=\underset{\sim}{0}$, we can define (cf. $[15, \S 2])$ the Airy stress function $\psi$ such that

$$
\begin{aligned}
& \frac{\partial^{2} \psi}{\partial x_{1}^{2}}=(2 \mu+\lambda) \frac{\partial u_{2}}{\partial x_{2}}+\lambda \frac{\partial u_{1}}{\partial x_{1}}, \\
& \frac{\partial^{2} \psi}{\partial x_{1} \partial x_{2}}=-\mu\left[\frac{\partial u_{1}}{\partial x_{2}}+\frac{\partial u_{2}}{\partial x_{1}}\right], \\
& \frac{\partial^{2} \psi}{\partial x_{2}^{2}}=(2 \mu+\lambda) \frac{\partial u_{1}}{\partial x_{1}}+\lambda \frac{\partial u_{2}}{\partial x_{2}},
\end{aligned}
$$

and

$$
\begin{array}{rlrl}
\Delta^{2} \psi & =0 & & \text { on } \Omega, \\
\left.\psi\right|_{\Gamma_{i}}=h_{i} & & \text { on } \Gamma_{i}, \\
\left.\frac{\partial \psi}{\partial \nu_{i}}\right|_{\Gamma_{i}}=k_{i} & & \text { on } \Gamma_{i} .
\end{array}
$$

Note that (cf. [15, §2] and [13, Theorem 1.5.2.8]) the conditions $\xi_{i}\left(S_{i+1}\right)=$

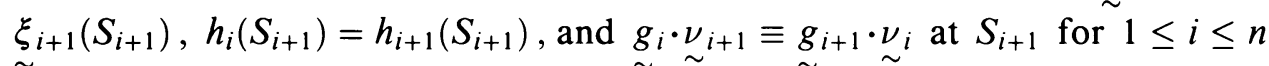
imply the existence of $\lambda \in H^{3}(\Omega)$ such that

$$
\left.\lambda\right|_{\Gamma_{i}}=h_{i},\left.\quad \frac{\partial \lambda}{\partial \nu_{i}}\right|_{\Gamma_{i}}=k_{i},
$$

and

$$
\|\lambda\|_{H^{3}(\Omega)} \leq C_{\Omega} \sum_{i=1}^{n}\left\{\left\|k_{i}\right\|_{H^{3 / 2}\left(\Gamma_{i}\right)}+\left\|h_{i}\right\|_{H^{5 / 2}\left(\Gamma_{i}\right)}\right\} .
$$

It follows from (2.42) and (2.43) that

$$
\begin{aligned}
\Delta^{2}(\psi-\lambda) & =-\Delta^{2} \lambda & & \text { on } \Omega, \\
\left.(\psi-\lambda)\right|_{\Gamma_{i}} & =0 & & \text { on } \Gamma_{i}, \\
\left.\frac{\partial(\psi-\lambda)}{\partial \nu_{i}}\right|_{\Gamma_{i}} & =0 & & \text { on } \Gamma_{i} .
\end{aligned}
$$


From the elliptic regularity of the biharmonic equation (cf. $[6,13])$ we have

$$
\|\psi-\lambda\|_{H^{3}(\Omega)} \leq C_{\Omega}\left\|\Delta^{2} \lambda\right\|_{H^{-1}(\Omega)},
$$

which in view of (2.40) and (2.44) implies that

$$
\|\psi\|_{H^{3}(\Omega)} \leq C_{\Omega} \sum_{i=1}^{n}\left\|\underset{\sim}{g_{i}}\right\|_{H^{1 / 2}\left(\Gamma_{i}\right)}
$$

From (2.41), we have

$$
\begin{aligned}
& 2(\mu+\lambda) \operatorname{div} \underset{\sim}{u}=\frac{\partial^{2} \psi}{\partial x_{1}^{2}}+\frac{\partial^{2} \psi}{\partial x_{2}^{2}}, \\
& \frac{\partial u_{1}}{\partial x_{1}}=\frac{(2 \mu+\lambda)}{4 \mu(\mu+\lambda)} \frac{\partial^{2} \psi}{\partial x_{2}^{2}}-\frac{\lambda}{4 \mu(\mu+\lambda)} \frac{\partial^{2} \psi}{\partial x_{1}^{2}}, \\
& \frac{\partial u_{2}}{\partial x_{2}}=\frac{(2 \mu+\lambda)}{4 \mu(\mu+\lambda)} \frac{\partial^{2} \psi}{\partial x_{1}^{2}}-\frac{\lambda}{4 \mu(\mu+\lambda)} \frac{\partial^{2} \psi}{\partial x_{2}^{2}}, \\
& \frac{1}{2}\left[\frac{\partial u_{1}}{\partial x_{2}}+\frac{\partial u_{2}}{\partial x_{1}}\right]=-\frac{1}{2 \mu} \frac{\partial^{2} \psi}{\partial x_{1} \partial x_{2}} .
\end{aligned}
$$

Combining (2.47) and (2.48), we obtain

$$
\lambda\|\operatorname{div} \underset{\sim}{u}\|_{H^{\prime}(\Omega)} \leq C_{\Omega} \sum_{i=1}^{n}\left\|{\underset{\sim}{i}}_{i}\right\|_{H^{1 / 2}\left(\Gamma_{i}\right)}
$$

and

$$
\|\underset{\sim}{\varepsilon} \underset{\sim}{(u)}\|_{H^{1}(\Omega)} \leq C_{\Omega} \sum_{i=1}^{n}\left\|\underset{\sim}{g_{i}}\right\|_{H^{1 / 2}\left(\Gamma_{i}\right)} .
$$

By Korn's second inequality (cf. (4.2) below) and Friedrichs' inequality (cf. [18]), inequality (2.50) implies that

$$
\|\underset{\sim}{u}\|_{H^{1}(\Omega)} \leq C_{\Omega} \sum_{i=1}^{n} \| \underset{\sim}{g}{\underset{\sim}{H^{1 / 2}\left(\Gamma_{i}\right)}} .
$$

Since the second-order derivatives of $u_{1}$ and $u_{2}$ are linear combinations of the

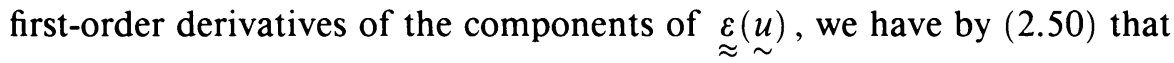

$$
|\underset{\sim}{\mid u}|_{H^{2}(\Omega)} \leq C_{\Omega} \sum_{i=1}^{n}\left\|\underset{\sim}{g_{i}}\right\|_{H^{1 / 2}\left(\Gamma_{i}\right)} .
$$

Inequality (2.30) now follows from (2.49), (2.51), and (2.52).

\section{THE PURE DISPLACEMENT PROBLEM}

We shall only consider the case of homogeneous boundary conditions, i.e., $\underset{\sim}{g_{i}}=\underset{\sim}{0}$ for $1 \leq i \leq n$. Our result can then be easily extended to the more general case where $\underset{\sim}{g_{i}} \in \underset{\sim}{H^{3 / 2}}\left(\Gamma_{i}\right), \sum_{i=1}^{n} \int_{\Gamma_{i}} \underset{\sim}{g_{i}} \cdot \underset{\sim}{\nu_{i}} d s=0$, and

$$
\frac{\partial}{\partial \tau_{i}} g_{i} \cdot \underset{\sim}{\nu_{i+1}} \equiv \frac{\partial}{\partial \tau_{i+1}} g_{\sim}+\nu_{\sim}
$$

at $S_{i+1}$ for $1 \leq i \leq n$. 
The boundary value problem (2.7) can be rewritten as:

$$
\begin{aligned}
-\mu \Delta \underset{\sim}{u}-(\mu+\lambda) \underset{\sim}{\operatorname{grad}(\operatorname{div} \underset{\sim}{u})}=\underset{\sim}{f} & \text { in } \Omega, \\
\underset{\sim}{u}=\underset{\sim}{0} & \text { on } \partial \Omega,
\end{aligned}
$$

where $f \in \underset{\sim}{L^{2}}(\Omega)$. It has the following weak formulation: Find $\underset{\sim}{u} \in \underset{\sim}{H_{0}^{1}}(\Omega)$ such that

$$
\mu \int_{\Omega} \underset{\approx}{\operatorname{grad}} \underset{\sim}{u}: \underset{\sim}{\operatorname{grad}} \underset{\sim}{v} d x+(\mu+\lambda) \int_{\Omega}(\operatorname{div} \underset{\sim}{u})(\operatorname{div} \underset{\sim}{v}) d x=\int_{\Omega} f \underset{\sim}{f} \cdot \underset{\sim}{v} d x
$$

for all $\underset{\sim}{v} \in \underset{\sim}{H_{0}^{1}}(\Omega)$.

By Poincaré's inequality, there exists a unique solution to problem (3.2), which is therefore the solution of (3.1). From Lemma 2.2, we know that the solution is actually in $\underset{\sim}{H^{2}}(\Omega) \cap \underset{\sim}{H_{0}^{1}}(\Omega)$.

In order to discretize (3.2), we introduce a triangulation $\mathscr{T}_{h}$. Here we let the subscript $h$ represent the maximum of the diameters of the triangles in the triangulation. Let

$$
\underset{\sim}{V_{h}}=\left\{\underset{\sim}{v}: \underset{\sim}{v} \in \underset{\sim}{L^{2}}(\Omega),\left.\underset{\sim}{v}\right|_{T} \text { is linear for all } T \in \mathscr{T}_{h},\right.
$$

$$
\underset{\sim}{v} \text { is continuous at the midpoints of interelement boundaries }
$$

$$
\text { and } \underset{\sim}{v}=\underset{\sim}{0} \text { at the midpoints of edges along } \partial \Omega\} \text {. }
$$

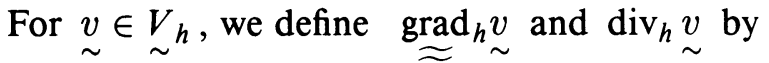

$$
\begin{aligned}
& \left.\left(\operatorname{grad}_{h} v\right)\right|_{T}=\underset{\approx}{\operatorname{grad}}\left(\left.\underset{\sim}{v}\right|_{T}\right), \\
& \left.\left(\operatorname{div}_{h} \underset{\sim}{v}\right)\right|_{T}=\operatorname{div}\left(\left.\underset{\sim}{v}\right|_{T}\right) \quad \forall T \in \mathscr{T}_{h} .
\end{aligned}
$$

The discretized problem is: Find $\underset{\sim}{u_{h}} \in \underset{\sim}{V_{h}}$ such that

$$
\begin{aligned}
& \mu \int_{\Omega} \underset{\operatorname{grad}_{h}}{\sim}{\underset{\sim}{h}}_{h}: \underset{\sim}{\operatorname{grad}_{h} v} \underset{\sim}{v} d x+(\mu+\lambda) \int_{\Omega}\left(\operatorname{div}_{h} \underset{\sim}{u_{h}}\right)\left(\operatorname{div}_{h} \underset{\sim}{v}\right) d x \\
& =\int_{\Omega} \underset{\sim}{f} \cdot \underset{\sim}{v} d x
\end{aligned}
$$

for all $\underset{\sim}{v} \in V_{h}$.

Let $\tilde{a}_{h}(\cdot, \cdot)$ be the symmetric positive definite bilinear form defined on $\underset{\sim}{V_{h}}+$ $\left.\underset{\sim}{H^{2}}(\Omega) \cap \underset{\sim}{H}(\Omega)\right)$ by

$$
\begin{aligned}
& a_{h}\left({\underset{\sim}{1}}_{1}, v_{\sim}\right)=\mu \int_{\Omega} \underset{\operatorname{grad}_{h}}{\approx}{\underset{\sim}{1}}_{1}:{\underset{\operatorname{grad}}{\approx}}_{h} v_{2} d x \\
& +(\mu+\lambda) \int_{\Omega}\left(\operatorname{div}_{h} \underset{\sim}{v_{1}}\right)\left(\operatorname{div}_{h} \underset{\sim}{v_{2}}\right) d x .
\end{aligned}
$$


Problem (3.6) can therefore be written as

$$
a_{h}\left(\underset{\sim}{u_{h}}, \underset{\sim}{v}\right)=\int_{\Omega} \underset{\sim}{f} \cdot \underset{\sim}{v} d x
$$

for all $\underset{\sim}{v} \in{\underset{\sim}{h}}_{h}$. By the positive definiteness of $a_{h}(\cdot, \cdot)$, equation (3.8) has a

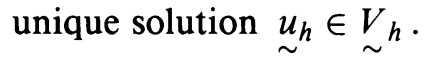

We define the nonconforming energy norm $\|\cdot\|_{h}$ on $\left.\underset{\sim}{V_{h}}+\underset{\sim}{H^{2}}(\Omega) \cap \underset{\sim}{H_{0}^{1}}(\Omega)\right)$ by

$$
\|\underset{\sim}{v}\|_{h}=a_{h}(\underset{\sim}{v}, \underset{\sim}{v})^{1 / 2}
$$

It is obvious that

$$
\underset{\sim}{\operatorname{grad}}{ }_{\sim} \underset{\sim}{v}\left\|_{L^{2}(\Omega)} \leq \mu_{0}^{-1 / 2}\right\| \underset{\sim}{v} \|_{h}
$$

There is an interpolation operator $\prod_{h}: \underset{\sim}{H^{2}}(\Omega) \cap \underset{\sim}{H_{0}^{1}}(\Omega) \rightarrow \underset{\sim}{V_{h}}$ defined by

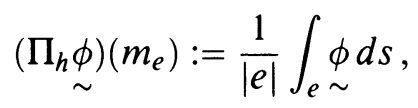

where $m_{e}$ is the midpoint of edge $e$. Then (cf. [9])

$$
\left.\operatorname{div}\left(\Pi_{h} \phi\right)\right|_{T}=\frac{1}{|T|} \int_{T} \operatorname{div} \underset{\sim}{\phi} d x \quad \forall T \in \mathscr{T}_{h},
$$

and

$$
\left\|\underset{\sim}{\phi}-\prod_{h} \phi\right\|_{L^{2}(\Omega)}+h \| \underset{\sim}{\operatorname{grad}_{h}\left(\phi-\prod_{h} \phi\right)} \underset{\sim}{\prod_{L^{2}(\Omega)} \leq C_{\theta} h^{2} \mid \underset{\sim}{\left.\phi\right|_{H^{2}(\Omega)}},}
$$

where $\theta$ is the minimum of all the angles in the triangulation $\mathscr{T}_{h}$.

Theorem 3.1. There exists a positive constant $C_{\Omega, \theta}$ such that

$$
\|\underset{\sim}{u}-\underset{\sim}{u} h\|_{h} \leq C_{\Omega, \theta} h\|\underset{\sim}{f}\|_{L^{2}(\Omega)} .
$$

Proof. Our analysis closely follows that of Crouzeix and Raviart for the Stokes problem in [9]. Let $\underset{\sim}{u_{h}^{*}}$ be the $a_{h}(\cdot, \cdot)$-orthogonal projection of $\underset{\sim}{u}$ into $\underset{\sim}{V_{h}}$. Following [19], we can deduce an abstract discretization error estimate:

$$
\begin{aligned}
& \left\|\underset{\sim}{u}-\underset{\sim}{u_{h}}\right\|_{h} \leq\left\|\underset{\sim}{u}-{\underset{\sim}{u}}_{h}^{*}\right\|_{h}+\left\|\underset{\sim}{u_{h}^{*}}-{\underset{\sim}{u}}_{h}\right\|_{h} \\
& \leq \inf _{v \in V_{\sim}}\|\underset{\sim}{u}-\underset{\sim}{v}\|_{h}+\sup _{\substack{v \in V_{\sim} \backslash\{0\} \\
\sim}} \frac{\left|a_{h}\left(\underset{\sim}{u_{h}^{*}}-\underset{\sim}{u_{h}}, \underset{\sim}{v}\right)\right|}{\|\underset{\sim}{v}\|_{h}} \\
& \leq \inf _{v \in V_{\sim}}\|\underset{\sim}{u}-\underset{\sim}{v}\|_{h}+\sup _{\substack{v \in V_{h} \backslash\{0\} \\
\sim}} \frac{\left|a_{h}(\underset{\sim}{u}, \underset{\sim}{v})-\int_{\Omega} f \underset{\sim}{f} \cdot \underset{\sim}{v} d x\right|}{\|\underset{\sim}{v}\|_{h}} .
\end{aligned}
$$


Using integration by parts, the weak continuity of $v$, and the BrambleHilbert lemma (cf. [7,9]), we have

$$
\begin{gathered}
\left|\int_{\Omega} \underset{\sim}{\underset{\sim}{\operatorname{grad}} u}: \underset{\sim}{\operatorname{grad}_{h}} \underset{\sim}{v} d x+\int_{\Omega} \underset{\sim}{\Delta u} \cdot \underset{\sim}{v} d x\right| \\
\leq C_{\Omega, \theta} h \mid \underset{\sim}{|u|_{H^{2}(\Omega)}} \| \underset{\sim}{\operatorname{grad}_{h} v\left\|_{\sim}\right\|_{L^{2}(\Omega)}}
\end{gathered}
$$

and

$$
\begin{gathered}
\left|\int_{\Omega} \underset{\operatorname{div}}{\underset{\sim}{u}} \operatorname{div}_{h} \underset{\sim}{v} d x+\int_{\Omega} \underset{\sim}{\operatorname{grad}(\operatorname{div} \underset{\sim}{u})} \cdot \underset{\sim}{v} d x\right| \\
\leq C_{\Omega, \theta} h \mid \operatorname{div} \underset{\sim}{\left.u\right|_{H^{1}(\Omega)}} \| \underset{\sim}{\operatorname{grad}_{h} \underset{\sim}{v} \|_{\mathcal{L}^{2}(\Omega)} .}
\end{gathered}
$$

Combining (2.8), (3.1), (3.7), (3.10), (3.16), and (3.17), we have

$$
\begin{aligned}
& \left|a_{h}(\underset{\sim}{u}, \underset{\sim}{v})-\int_{\Omega} \underset{\sim}{f} \cdot \underset{\sim}{v} d x\right| \\
& \leq C_{\Omega, \theta} h\left\|\underset{\sim}{\operatorname{grad}_{h}} \underset{\sim}{v}\right\|_{L^{2}(\Omega)}\left\{\mu \underset{\sim}{\mu|u|_{H^{2}(\Omega)}}+(\mu+\lambda)|\operatorname{div} \underset{\sim}{u}|_{H^{1}(\Omega)}\right\} \\
& \leq C_{\Omega, \theta} h\|\underset{\sim}{v}\|_{h}\left\|f_{\sim}\right\|_{L^{2}(\Omega)} .
\end{aligned}
$$

By Lemma 2.1 there exists $\underset{\sim}{u_{1}} \in \underset{\sim}{H^{2}}(\Omega) \cap \underset{\sim}{H_{0}^{1}}(\Omega)$ such that

$$
\operatorname{div} \underset{\sim}{u_{1}}=\operatorname{div} \underset{\sim}{u}
$$

and

$$
\|\underset{\sim}{u}\|_{H^{2}(\Omega)} \leq C_{\Omega}\|\operatorname{div} \underset{\sim}{\underset{\sim}{u}}\|_{H^{1}(\Omega)} .
$$

Combining (2.8) and (3.20), we have

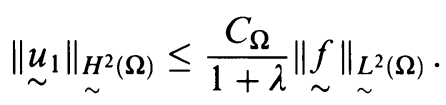

Note that (3.12) and (3.19) imply that

$$
\operatorname{div}_{h} \Pi_{h} \underset{\sim}{u_{1}}=\operatorname{div}_{h} \Pi_{h} \underset{\sim}{u} .
$$

Using (2.8), (3.7), (3.9), (3.13), (3.19), (3.21), and (3.22), we obtain

$$
\begin{aligned}
& \inf _{v \in V_{h}}\left(\|\underset{\sim}{u}-\underset{\sim}{v}\|_{h}\right) \leq\left\|\underset{\sim}{u}-\Pi_{h} \underset{\sim}{u}\right\|_{h} \\
& =\left(\mu\left\|\underset{\sim}{\sim \operatorname{grad}_{h}}\left(\underset{\sim}{u}-\prod_{h} \underset{\sim}{u}\right)\right\|_{L^{2}(\Omega)}^{2}+(\mu+\lambda)\left\|\operatorname{div}_{h}\left(\underset{\sim}{u_{1}}-\Pi_{h} \underset{\sim}{u_{1}}\right)\right\|_{L^{2}(\Omega)}^{2}\right)^{1 / 2} \\
& \leq C_{\Omega, \theta} h\|\underset{\sim}{f}\|_{L^{2}(\Omega)}
\end{aligned}
$$

The theorem now follows by combining (3.15), (3.18), and (3.23). 
Theorem 3.2. There exists a positive constant $C_{\Omega, \theta}$ such that

$$
\|\underset{\sim}{u}-\underset{\sim}{u}\|_{L^{2}(\Omega)} \leq C_{\Omega, \theta} h^{2}\|\underset{\sim}{f}\|_{L^{2}(\Omega)} .
$$

Proof. The proof is based on a duality argument. We have

$$
\|\underset{\sim}{u}-\underset{\sim}{u}\|_{L^{2}(\Omega)}=\sup _{\substack{w \in L^{2}(\Omega) \backslash\{0\} \\ \sim}} \frac{\left|\int_{\Omega}\left(\underset{\sim}{u}-\underset{\sim}{u} u_{h}\right) \cdot \underset{\sim}{w} d x\right|}{\|\underset{\sim}{w}\|_{L^{2}(\Omega)}} .
$$

Given $\underset{\sim}{w} \in \underset{\sim}{L^{2}}(\Omega)$, let $\underset{\sim}{\zeta} \in \underset{\sim}{H^{2}}(\Omega) \cap \underset{\sim}{H}(\Omega)$ satisfy

$$
\begin{aligned}
-\mu \Delta \underset{\sim}{\zeta}-(\mu+\lambda) \underset{\sim}{\operatorname{grad}(\operatorname{div} \underset{\sim}{\zeta})}=\underset{\sim}{w} \text { in } \Omega, \\
\underset{\sim}{\zeta}=\underset{\sim}{0} \text { on } \partial \Omega
\end{aligned}
$$

and $\underset{\sim}{\zeta_{h}} \in \underset{\sim}{V_{h}}$ satisfy

$$
a_{h}\left(\underset{\sim}{\zeta_{h}}, \underset{\sim}{v}\right)=\int_{\Omega} \underset{\sim}{w} \cdot \underset{\sim}{v} d x \quad \underset{\sim}{\forall} \in \underset{\sim}{V}
$$

By Lemma 2.2 we have the estimate

$$
\left\|\underset{\sim}{\|_{H^{2}(\Omega)}}+\lambda\right\| \operatorname{div} \underset{\sim}{\zeta}\left\|_{H^{1}(\Omega)} \leq C_{\Omega}\right\| \underset{\sim}{w} \|_{L^{2}(\Omega)}
$$

By Theorem 3.1 we also have

$$
\|\underset{\sim}{\zeta}-\underset{\sim}{\zeta}\|_{h} \leq C_{\Omega, \theta} h\|\underset{\sim}{w}\|_{L^{2}(\Omega)}
$$

Using (3.27), the Cauchy-Schwarz inequality, and (3.8), we have

$$
\begin{aligned}
& \left|\int_{\Omega}\left(\underset{\sim}{u}-\underset{\sim}{u_{h}}\right) \cdot \underset{\sim}{w} d x\right|=\left|a_{h}(\underset{\sim}{\zeta}, \underset{\sim}{u})-a_{h}\left(\underset{\sim}{\zeta_{h}}, \underset{\sim}{u_{h}}\right)\right| \\
& =\mid a_{h}\left(\underset{\sim}{\zeta}-{\underset{\sim}{\zeta}}_{h}, \underset{\sim}{u}-\prod_{h} \underset{\sim}{u}\right)+a_{h}\left(\underset{\sim}{\zeta}-\sim_{\sim}^{\zeta_{h}}, \prod_{h} \underset{\sim}{u}\right) \\
& +a_{h}\left(\underset{\sim}{\zeta_{h}}-\prod_{h} \underset{\sim}{\zeta}, \underset{\sim}{u}-\underset{\sim}{u_{h}}\right)+a_{h}\left(\Pi_{h} \underset{\sim}{\zeta} \underset{\sim}{u}-\underset{\sim}{u} \sim_{h}\right) \mid \\
& \leq\left\|\underset{\sim}{\zeta}-\underset{\sim}{\zeta_{h}}\right\|_{h}\left\|\underset{\sim}{u}-\Pi_{h} \underset{\sim}{u}\right\|_{h}+\left\|\underset{\sim}{\zeta_{h}}-\pi_{h} \zeta\right\|_{h}\|\|_{\sim}^{u}-{\underset{\sim}{u}}_{h} \|_{h} \\
& +\left|a_{h}\left(\underset{\sim}{\zeta}-\underset{\sim}{\zeta_{h}}, \prod_{h} \underset{\sim}{u}\right)\right|+\left|a_{h}\left(\prod_{h} \underset{\sim}{\zeta}, \underset{\sim}{u}-\underset{\sim}{u}\right)\right| .
\end{aligned}
$$
that

Note that (3.14), (3.28), (3.29), and the argument that led to (3.23) imply (3.31) $\underset{\sim}{\zeta}-\underset{\sim}{\zeta}\left\|_{h}\right\| \underset{\sim}{u}-\prod_{h} \underset{\sim}{u}\left\|_{h}+\right\| \underset{\sim}{\zeta_{h}}-\prod_{h} \underset{\sim}{\zeta}\left\|_{h}\right\| \underset{\sim}{u}-\underset{\sim}{u}\left\|_{h} \leq C_{\Omega, \theta} h^{2}\right\| \underset{\sim}{w}\left\|_{L^{2}(\Omega)}\right\| \underset{\sim}{f} \|_{L^{2}(\Omega)}$. It remains to estimate $\left|a_{h}\left(\prod_{h} \underset{\sim}{\zeta}, \underset{\sim}{u}-\underset{\sim}{u} u_{h}\right)\right|=\left|a_{h}\left(\prod_{h} \underset{\sim}{\zeta}, \underset{\sim}{u}\right)-\int_{\Omega} f \cdot\left(\prod_{h} \zeta\right) d x\right|$ and $\left|a_{h}\left(\underset{\sim}{\zeta}-\underset{\sim}{\zeta}, \Pi_{h} \underset{\sim}{u}\right)\right|=\left|a_{h}\left(\underset{\sim}{\zeta}, \Pi_{h} \underset{\sim}{u}\right)-\int_{\Omega} \underset{\sim}{w} \cdot \Pi_{h} \underset{\sim}{u} d x\right|$.

Using integration by parts, the weak continuity of $\Pi_{h} \zeta$, and the BrambleHilbert lemma (cf. [9]), we have

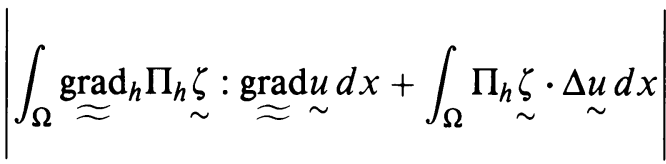

$$
\begin{aligned}
& \leq C_{\Omega, \theta} h^{2} \mid \underset{\sim}{\dot{j}_{H^{2}(\Omega)}}{\left.\underset{\sim}{u}\right|_{H^{2}(\Omega)}}
\end{aligned}
$$


and

$$
\begin{aligned}
& \left|\int_{\Omega}\left(\operatorname{div}_{h} \prod_{h} \zeta\right)(\operatorname{div} \underset{\sim}{u}) d x+\int_{\Omega} \prod_{h} \underset{\sim}{\zeta} \cdot \underset{\sim}{\operatorname{grad}}(\operatorname{div} \underset{\sim}{v}) d x\right|
\end{aligned}
$$

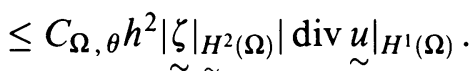

Combining (2.8), (3.1), (3.7), (3.28), (3.31), (3.32), and (3.33), we have

$$
\begin{aligned}
& \left|a_{h}\left(\prod_{h} \underset{\sim}{\zeta}, \underset{\sim}{u}\right)-\int_{\Omega} \underset{\sim}{f} \cdot\left(\prod_{h} \zeta\right) d x\right| \\
& \quad \leq C_{\Omega, \theta} h^{2} \mid \underset{\sim}{\left.\zeta\right|_{H^{2}(\Omega)}}\left\{\underset{\sim}{\mu|\underset{\sim}{\mid}|_{H^{2}(\Omega)}}+(\mu+\lambda)|\operatorname{div} \underset{\sim}{u}|_{H^{1}(\Omega)}\right\} \\
& \quad \leq C_{\Omega, \theta} h^{2}\|\underset{\sim}{w}\|_{L^{2}(\Omega)}\|\underset{\sim}{f}\|_{L^{2}(\Omega)} .
\end{aligned}
$$

Similarly, we have

$$
\left|a_{h}\left(\zeta-\zeta_{\sim}, \prod_{h} \underset{\sim}{u}\right)\right| \leq C_{\Omega, \theta} h^{2}\|\underset{\sim}{w}\|_{L^{2}(\Omega)}\|\underset{\sim}{f}\|_{L^{2}(\Omega)} .
$$

It follows from (3.30), (3.31), (3.34), and (3.35) that

$$
\left|\int_{\Omega}(\underset{\sim}{u}-\underset{\sim}{u} h) \cdot \underset{\sim}{w} d x\right| \leq C_{\Omega, \theta} h^{2}\|\underset{\sim}{w}\|_{L^{2}(\Omega)}\left\|\sim_{\sim}^{f}\right\|_{L^{2}(\Omega)} .
$$

The theorem now follows from (3.25) and (3.36).

\section{THE PURE TRACTION PROBLEM}

We assume here that all the assumptions in Lemma 2.3 hold. To simplify notation, we shall write the sum $\sum_{i=1}^{n} \int_{\Gamma_{i}} \underset{\sim}{g_{i}} \cdot \underset{\sim}{v} d s$ as $\int_{\partial \Omega} \underset{\sim}{g} \cdot \underset{\sim}{v} d s$. The boundary

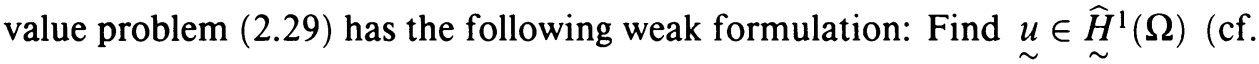
(1.3)) such that

$$
\begin{gathered}
\left.2 \mu \int_{\Omega} \underset{\approx}{\varepsilon} \underset{\sim}{u}\right): \underset{\approx}{\varepsilon}(v) d x+\lambda \int_{\Omega}(\operatorname{div} \underset{\sim}{u})(\operatorname{div} \underset{\sim}{v}) d x \\
=\int_{\Omega} f \cdot \underset{\sim}{v} d x+\int_{\partial \Omega} g \underset{\sim}{v} d s
\end{gathered}
$$

for all $\underset{\sim}{v} \in \widehat{\sim}^{1}(\Omega)$.

The following Korn's second inequality is well known (cf. [10, 12, 18]): There exists a positive constant $C_{\Omega}$ such that

$$
\|\underset{\sim}{\varepsilon}(\underset{\sim}{v})\|_{L^{2}(\Omega)} \geq C_{\Omega}|\underset{\sim}{v}|_{H^{1}(\Omega)} \quad \forall \underset{\sim}{v} \in \underset{\sim}{\widehat{H}^{1}}(\Omega) .
$$

In view of inequality (4.2), problem (4.1) has a unique solution, which is the solution of (2.29). From Lemma 2.3, we know that the solution is actually in $\widehat{H}^{2}(\Omega)$.

Let $\mathscr{T}_{h}$ be a triangulation obtained by connecting the midpoints of edges in the triangulation $\mathscr{T}_{2 h}$. Define

$$
\widehat{\sim}_{h}=\left\{\underset{\sim}{v} \in \underset{\sim}{\widehat{H}^{1}}(\Omega):\left.\underset{\sim}{v}\right|_{T} \text { is linear for all } T \in \mathscr{T}_{h}\right\}
$$


and

$$
M_{2 h}=\left\{v \in L^{2}(\Omega):\left.v\right|_{T} \text { is a constant for all } T \in \mathscr{T}_{2 h}\right\} .
$$

Let $P_{0}$ be the orthogonal projection from $L^{2}(\Omega)$ onto $M_{2 h}$, i.e., for $w \in L^{2}(\Omega)$

$$
\int_{\Omega}\left(P_{0} w\right) d x=\int_{\Omega} w v d x \quad \forall v \in M_{2 h} .
$$

The discretized problem, introduced by Falk in [10] (where a uniform convergence result based on a mixed formulation was outlined), is: Find $\underset{\sim}{u_{h}} \in{\underset{\sim}{W}}_{h}$ such that

$$
\begin{aligned}
\left.2 \mu \int_{\Omega} \underset{\sim}{\varepsilon} \underset{\sim}{\left(u_{h}\right.}\right): \underset{\approx}{\varepsilon}(\underset{\sim}{v}) d x+\lambda \int_{\Omega}\left(P_{0} \operatorname{div} \underset{\sim}{u_{h}}\right)\left(P_{0} \operatorname{div} \underset{\sim}{v}\right) d x \\
=\int_{\Omega} \underset{\sim}{f} \cdot \underset{\sim}{v} d x+\int_{\partial \Omega} g \underset{\sim}{v} d s
\end{aligned}
$$

for all $\underset{\sim}{v} \in \widehat{W}_{h}$.

The discretized problem (4.6) is an example of the method of reduced integration. We refer the readers to [17] for more information on this technique.

Let $a_{h}(\cdot, \cdot)$ be the symmetric bilinear form defined on $\underset{\sim}{\widehat{H}^{1}}(\Omega)$ by

$$
a_{h}\left(\underset{\sim}{v_{1}}, \underset{\sim}{v_{2}}\right)=2 \mu \int_{\Omega} \underset{\approx}{\varepsilon}\left(\underset{\sim}{v_{1}}\right): \underset{\approx}{\varepsilon}\left(v_{\sim}^{v_{2}}\right) d x+\lambda \int_{\Omega}\left(P_{0} \operatorname{div} \underset{\sim}{v_{1}}\right)\left(P_{0} \operatorname{div} \underset{\sim}{v_{2}}\right) d x .
$$

Problem (4.6) can be rewritten as

$$
a_{h}\left(\underset{\sim}{u_{h}}, \underset{\sim}{v}\right)=\int_{\Omega} f \cdot \underset{\sim}{v} d x+\int_{\partial \Omega} \underset{\sim}{g} \cdot \underset{\sim}{v} d x \quad \forall \underset{\sim}{\forall} \in \underset{\sim}{\widehat{W}_{h}} .
$$

By $(4.2), a_{h}(\cdot, \cdot)$ is positive definite. Hence (4.8) has a unique solution $\underset{\sim}{u_{h}} \in$ $\widehat{W}_{h}$.

We define the $\left(\mathscr{T}_{h}\right.$-dependent $)$ energy norm $\|\cdot\|_{h}$ on $\underset{\sim}{\widehat{H}}{ }^{1}(\Omega)$ by

$$
\|\underset{\sim}{v}\|_{h}=a_{h}(\underset{\sim}{v}, \underset{\sim}{v})^{1 / 2}
$$

It is obvious from (4.2) that there exists a positive constant $C_{\Omega}$ such that

$$
\left.\underset{\sim}{v}\right|_{H^{1}(\Omega)} \leq C_{\Omega}\|\underset{\sim}{v}\|_{h}
$$

Let $J_{h}: \underset{\sim}{H^{2}}(\Omega) \rightarrow \underset{\sim}{W_{h}}:=\left\{\underset{\sim}{v} \in \underset{\sim}{H^{1}}(\Omega):\left.\underset{\sim}{v}\right|_{T}\right.$ is linear for all $\left.T \in \mathscr{T}_{h}\right\}$ be defined in the following way. Let $p$ be a vertex of $\mathscr{T}_{h}$. If $p$ is also a vertex of $\mathscr{T}_{2 h}$, then

$$
\underset{\sim}{\left(J_{h} \phi\right)}(p)=\underset{\sim}{\phi}(p)
$$

Otherwise, there exist two vertices $p_{1}$ and $p_{2}$ of $\mathscr{T}_{2 h}$ such that $p$ is the midpoint of the edge $e$ connecting $p_{1}$ and $p_{2}$. Then

$$
\left(J_{h} \phi\right)(p)=\frac{2}{|e|} \int_{e} \phi d s-\frac{\underset{\sim}{\sim}\left(p_{1}\right)+\phi\left(p_{2}\right)}{2} .
$$


By the divergence and curl theorems and the fact that $J_{h} \phi$ is piecewise linear, it is immediate that

$$
\int_{T} \operatorname{div}\left(J_{h} \phi\right) d x=\int_{T} \operatorname{div} \underset{\sim}{\phi} d x
$$

and

$$
\int_{T} \operatorname{rot}\left(J_{h} \phi\right) d x=\int_{T} \operatorname{rot} \underset{\sim}{\phi} d x
$$

for all $T \in \mathscr{T}_{2 h}$.

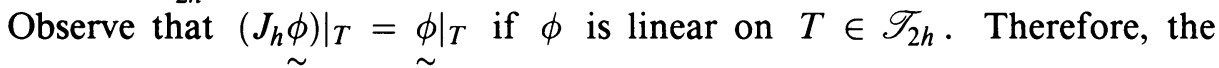
Bramble-Hilbert lemma and a homogeneity argument imply that there exists a positive constant $C_{\theta}$ such that

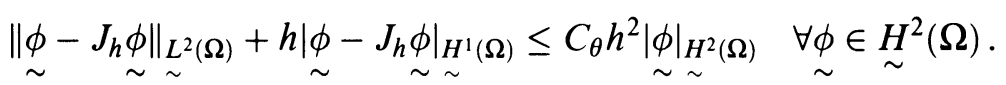

Here, $\theta$ is again the minimum angle in $\mathscr{T}_{h}$.

Let the interpolation operator $\Pi_{h}$ be defined by

$$
\Pi_{h} \phi:=\underset{\sim}{J_{h} \phi}-\frac{1}{|\Omega|} \int_{\Omega} J_{h} \phi d x .
$$

Clearly, $\int_{\Omega} \Pi_{h} \phi d x=0$, and in view of (4.14), $\int_{\Omega} \operatorname{rot}\left(\Pi_{h} \phi\right) d x=0$ if $\int_{\Omega} \operatorname{rot} \phi=$ 0 . Therefore, $\Pi_{h}$ maps $\underset{\sim}{\widehat{H}^{2}}(\Omega)$ into $\underset{\sim}{\widehat{W}} h$.

From (4.13) we have

$$
P_{0} \operatorname{div} \prod_{h} \underset{\sim}{\phi}=P_{0} \operatorname{div} \underset{\sim}{\phi} \underset{\sim}{\forall \phi \in \underset{\sim}{\widehat{H}^{2}}(\Omega)} .
$$

It follows easily from (4.15) that

$$
\begin{aligned}
\| \phi- & \underset{\sim}{\prod_{h} \phi \|_{L^{2}(\Omega)}}+h \mid \underset{\sim}{\phi}-\underset{\sim}{\left.\prod_{h} \phi\right|_{H^{1}(\Omega)}} \\
& \leq C_{\Omega, \theta} h^{2} \mid \underset{\sim}{\left.\right|_{H^{2}(\Omega)}} \quad \underset{\sim}{\forall \in} \underset{\sim}{\widehat{\sim}^{2}}(\Omega) .
\end{aligned}
$$

Theorem 4.1. There exists a positive constant $C_{\Omega, \theta}$ such that

$$
\left\|\underset{\sim}{u}-\underset{\sim}{u_{h}}\right\|_{h} \leq C_{\Omega, \theta} h\left\{\|\underset{\sim}{\| f}\|_{L^{2}(\Omega)}+\sum_{i=1}^{n} \| \underset{\sim}{g_{i} \|_{H^{1 / 2}\left(\Gamma_{i}\right)}}\right\} .
$$

Proof. The proof of the following abstract discretization error estimate is the same as that of inequality (3.15):

$$
\left\|\underset{\sim}{u}-\underset{\sim}{u_{h}}\right\|_{h}=\inf _{\substack{v \in V_{h} \\ \sim}}\|\underset{\sim}{u}-\underset{\sim}{v}\|_{h}
$$

$$
+\sup _{\substack{v \in V_{h} \backslash\{0\} \\ \sim}} \frac{\left|a_{h}(\underset{\sim}{u}, \underset{\sim}{v})-\int_{\Omega} \underset{\sim}{f} \cdot \underset{\sim}{v} d x-\int_{\partial \Omega} g \underset{\sim}{g} \cdot \underset{\sim}{v} d x\right|}{\|\underset{\sim}{v}\|_{h}} .
$$


Using (2.30), (4.1), (4.7), (4.10), and standard interpolation error estimates (cf. [8]), we have for $\underset{\sim}{v} \in \widehat{\sim}_{h} \subseteq{\underset{\sim}{H}}^{1}(\Omega)$

$$
\begin{aligned}
& \left|a_{h}(\underset{\sim}{u} \underset{\sim}{v})-\int_{\Omega} \underset{\sim}{f} \cdot \underset{\sim}{v} d x-\int_{\partial \Omega} \underset{\sim}{g} \cdot \underset{\sim}{v} d s\right| \\
& \left.=\mid 2 \mu \int_{\Omega} \underset{\sim}{\varepsilon} \underset{\sim}{u}\right): \underset{\approx}{\varepsilon}(\underset{\sim}{v}) d x+\lambda \int_{\Omega}\left(P_{0} \operatorname{div} \underset{\sim}{u}\right)(\operatorname{div} \underset{\sim}{v}) d x \\
& -\int_{\Omega} \underset{\sim}{f} \cdot \underset{\sim}{v} d x-\int_{\partial \Omega} \underset{\sim}{g} \cdot \underset{\sim}{v} d s \mid \\
& =\left|\lambda \int_{\Omega}\left(P_{0} \operatorname{div} \underset{\sim}{u}-\operatorname{div} \underset{\sim}{u}\right)(\operatorname{div} \underset{\sim}{v}) d x\right| \\
& \leq \lambda\left\|P_{0} \operatorname{div} \underset{\sim}{u}-\operatorname{div} \underset{\sim}{u}\right\|_{L^{2}(\Omega)}\|\operatorname{div} \underset{\sim}{v}\|_{L^{2}(\Omega)} \\
& \leq \lambda C_{\Omega, \theta} h\left|\operatorname{div} \underset{\sim}{\left.u\right|_{H^{1}(\Omega)}}\right| \underset{\sim}{\left.v\right|_{H^{1}(\Omega)}} \\
& \leq C_{\Omega, \theta} h\left\{\|\underset{\sim}{f}\|_{\sim}{\underset{\sim}{2}(\Omega)}+\sum_{i=1}^{n}\left\|\underset{\sim}{g_{i}}\right\|_{H^{1 / 2}\left(\Gamma_{i}\right)}\right\}\|\underset{\sim}{v}\|_{h} .
\end{aligned}
$$

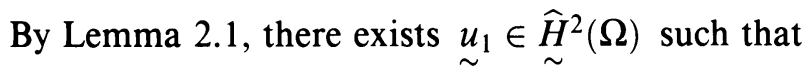

$$
\operatorname{div} \underset{\sim}{u_{1}}=\operatorname{div} \underset{\sim}{u}
$$

and

$$
\left\|\underset{\sim}{u_{1}}\right\|_{H^{2}(\Omega)} \leq C_{\Omega}\|\operatorname{div} \underset{\sim}{u}\|_{H^{1}(\Omega)} .
$$

Combining (2.30) and (4.23), we have

$$
\left\|\underset{\sim}{u_{1}}\right\|_{H^{2}(\Omega)} \leq \frac{C_{\Omega}}{1+\lambda}\left\{\|\underset{\sim}{f}\|_{L^{2}(\Omega)}+\sum_{i=1}^{n} \underset{\sim}{\|\underset{\sim}{g}\|_{H^{1 / 2}\left(\Gamma_{i}\right)}}\right\} .
$$

Note that (4.17) and (4.22) imply that

$$
P_{0} \operatorname{div} \Pi_{h} \underset{\sim}{u_{1}}=P_{0} \operatorname{div} \Pi_{h} \underset{\sim}{u} \text {. }
$$

Combining (2.30), (4.7), (4.9), (4.18), (4.22), (4.24), and (4.25), we obtain

$$
\begin{aligned}
& \inf _{v \in \widehat{W}_{h}}\|\underset{\sim}{u}-\underset{\sim}{v}\|_{h} \leq\left\|\underset{\sim}{u}-\Pi_{h} \underset{\sim}{u}\right\|_{h} \\
& \left.=\left\{2 \mu \int_{\Omega} \underset{\sim}{\underset{\sim}{\varepsilon}} \underset{\sim}{u}-\prod_{h} \underset{\sim}{u}\right): \underset{\approx}{\varepsilon} \underset{\sim}{u}-\prod_{h} \underset{\sim}{u}\right) d x \\
& \left.\left.+\lambda \int_{\Omega}\left(P_{0} \operatorname{div} \underset{\sim}{u}-\prod_{h} \underset{\sim}{u}\right)\right)^{2} d x\right\}^{1 / 2} \\
& \left.\leq\left\{2 \mu \underset{\sim}{u}-\left.\prod_{h} \underset{\sim}{u}\right|_{H^{1}(\Omega)} ^{2}+\lambda\left\|\operatorname{div}\left(\underset{\sim}{u_{1}}-\Pi_{h} \underset{\sim}{u_{1}}\right)\right\|_{L^{2}(\Omega)}^{2}\right)\right\}^{1 / 2} \\
& \leq\left\{C_{\Omega, \theta} h^{2}\left(\underset{\sim}{\mid \underset{H^{2}(\Omega)}{u}}+\lambda \underset{\sim}{\left.\operatorname{\sim }_{1}\right|_{H^{2}(\Omega)} ^{2}}\right)\right\}^{1 / 2} \\
& \leq C_{\Omega, \theta} h\left\{\|\underbrace{}_{\sim}\|_{L^{2}(\Omega)}+\sum_{i=1}^{n}\left\|\underset{\sim}{g_{i}}\right\|_{H^{1 / 2}\left(\Gamma_{i}\right)}\right\} .
\end{aligned}
$$

The theorem now follows from (4.20), (4.21), and (4.26). 


\section{BIBLIOGRAPHY}

1. D. N. Arnold, F. Brezzi, and J. Douglas, Jr., PEERS: A new mixed finite element for plane elasticity, Japan J. Appl. Math. 1 (1984), 347-367.

2. D. N. Arnold, L. R. Scott, and M. Vogelius, Regular inversion of the divergence operator with Dirichlet boundary conditions on a polygon, Ann. Scuola Norm. Sup. Pisa Cl. Sci. (4) 15 (1988), 169-192.

3. I. Babuška and M. Suri, On locking and robustness in the finite element method, preprint.

4. __ Locking effects in the finite element approximation of elasticity problems, preprint.

5. I. Babuška and B. Szabo, On the rates of convergence of the finite element method, Internat. J. Numer. Meth. Engrg. 18 (1982), 323-341.

6. H. Blum and R. Rannacher, On the boundary value problem of the biharmonic operator on domains with angular corners, Math. Methods Appl. Sci. 2 (1980), 556-581.

7. J. H. Bramble and S. R. Hilbert, Estimation of linear functionals on Sobolev spaces with application to Fourier transforms and spline interpolation, SIAM J. Numer. Anal. 7 (1970), 113-124.

8. P. G. Ciarlet, The finite element method for elliptic problems, North-Holland, AmsterdamNew York-Oxford, 1978.

9. M. Crouzeix and P.-A. Raviart, Conforming and nonconforming finite element methods for solving the stationary Stokes equations. I, RAIRO R-3 (1973), 33-75.

10. R. S. Falk, Nonconforming finite element methods for the equations of linear elasticity, Math. Comp. 57 (1991), 529-550.

11. L. Franca and R. Stenberg, Error analysis of some Galerkin-least-squares methods for the elasticity equations, preprint.

12. V. Girault and P.-A. Raviart, Finite element methods for Navier-Stokes equations, SpringerVerlag, Berlin, Heidelberg, 1986.

13. P. Grisvard, Elliptic problems in nonsmooth domains, Pitman, Boston, 1985.

14. __ Problèmes aux limites dans les polygones. Mode d'emploi, EDF Bull. Direction Études Rech. Sér. C. Math. Inform. 1 (1986), 21-59.

15. Singularités en elasticité, Arch. Rational Mech. Anal. 107 (1989), 157-180.

16. R. B. Kellogg and J. E. Osborn, A regularity result for the Stokes problem on a convex polygon, J. Funct. Anal. 21 (1976), 397-431.

17. D. S. Malkus and T. J. R. Hughes, Mixed finite element methods-reduced and selective integration techniques: A unification of concepts, Comput. Methods Appl. Mech. Engrg. 15 (1978), 63-81.

18. J. Nečas, Les méthodes directes en théorie des équations elliptiques, Masson, Paris, 1967.

19. R. Scott, Interpolated boundary conditions in the finite element method, SIAM J. Numer. Anal. 12 (1975), 404-427.

20. R. Stenberg, A family of mixed finite elements for the elasticity problem, Numer. Math. 53 (1988), 513-538.

21. T. Valent, Boundary value problems of finite elasticity, Springer-Verlag, New York, 1988.

22. M. Vogelius, An analysis of the p-version of the finite element method for nearly incompressible materials. Uniformly valid, optimal error estimates, Numer. Math. 41 (1983), 39-53.

Department of Mathematics and Computer Science, Clarkson University, Potsdam, NEW YORK 13699-5815

E-mail address: brenner@sun.mcs.clarkson.edu

E-mail address: sung@sun.mcs.clarkson.edu 